\title{
Benchmark-Driven Algorithm Configuration Applied to Parallel Model-Based Optimization
}

This paper was downloaded from TechRxiv (https://www.techrxiv.org).

\section{LICENSE}

CC BY 4.0

SUBMISSION DATE / POSTED DATE

24-01-2022 / 28-01-2022

\section{CITATION}

Rehbach, Frederik; Zaefferer, Martin; Fischbach, Andreas; Rudolph, Günter; Bartz-Beielstein, Thomas (2022): Benchmark-Driven Algorithm Configuration Applied to Parallel Model-Based Optimization. TechRxiv. Preprint. https://doi.org/10.36227/techrxiv.18999767.v1

$\mathrm{DOI}$

10.36227/techrxiv.18999767.v1 


\title{
Benchmark-Driven Algorithm Configuration Applied to Parallel Model-Based Optimization
}

\author{
Frederik Rehbach, Martin Zaefferer, Andreas Fischbach, Günter Rudolph, Thomas Bartz-Beielstein
}

\begin{abstract}
This paper introduces a benchmarking framework that allows rigorous evaluation of parallel model-based optimizers for expensive functions. The framework establishes a relationship between estimated costs of parallel function evaluations (on real-world problems) to known sets of test functions. Such real-world problems are not always readily available (e.g., confidentiality, proprietary software). Therefore, new test problems are created by Gaussian process simulation. The proposed framework is applied in an extensive benchmark study to compare multiple state-of-the-art parallel optimizers with a novel model-based algorithm, which combines ideas of an explorative search for global model quality with parallel local searches to increase function exploitation. The benchmarking framework is used to configure good batch size setups for parallel algorithms systematically based on landscape properties. Furthermore, we introduce a proof-of-concept for a novel automatic batch size configuration. The predictive quality of the batch size configuration is evaluated on a large set of test functions and the functions generated by Gaussian process simulation. The introduced algorithm outperforms multiple state-of-the-art optimizers, especially on multi-modal problems. Additionally, it proves to be particularly robust over various problem landscapes, and performs well with all tested batch sizes. Consequently, this makes it well-suited for black-box kinds of problems.
\end{abstract}

Index Terms-Benchmarking, Exploratory Landscape Analysis, Model-Based Optimization, Parallelization, Simulation

\section{INTRODUCTION}

$\mathbf{F}$ OR many optimization problems, a significant amount of resources is required for each objective function evaluation. For example, the cost per evaluation might be attributed to the time an employee requires to perform experiments, material costs, or computational cost of computer experiments such as Computational Fluid Dynamics (CFD) simulations. In such an environment, optimizers are often limited to a few tens or hundreds of function evaluations. Among others, two regularly applied methods to cope with expensive-to-evaluate problems are surrogate model-based optimization (SBO) and parallelization.

In SBO a data-driven surrogate model is fitted to known sample points. The surrogate replaces the expensive-toevaluate objective function, enabling an optimizer to search for the model's optimum extensively. The search is led by an infill criterion that judges the quality of a given point on the model. Such an infill criterion might be the model prediction itself or other criteria such as the model's uncertainty. Kriging (also called Gaussian process regression) is commonly applied in SBO as it is argued to deliver high predictive quality, even when only a few sample points are given, as well as providing an intrinsic estimate of its model uncertainty [12]. Expected improvement (EI) is known as an infill criterion from its implementation in EGO by Jones et al. [20]. It is supposed to combine exploration and exploitation to efficiently guide the search on the model by using Kriging's uncertainty estimate. The best point found according to the infill criterion is expensively evaluated on the objective function, and the model is updated with the new information. This process is iteratively repeated until a termination criterion is reached.

Even though model-based optimization shows success in many practical applications [29], [3], it is a sequential approach and not yet parallel in its most basic form. There exist infill criteria such as q-point expected improvement (QEI) that search for a set of points on the model rather than a single candidate, allowing for a parallel evaluation of the proposed set of points. Q-EI is the theoretical extension of EI from a single point to the expected improvement of a set of points. The method, as introduced by Schonlau [35], was further adapted by Ginsbourger et al. [13] for a more computationally efficient implementation. In recent research, it is often argued that these methods lead to too much search space exploration and thus do not make efficient use of the limited evaluation budget anymore [33], [38]. Wessing and Preuss argue that the theoretical convergence proof of EI has little influence on practical applications. Under the severely limited evaluation budget, the asymptotic convergence behavior of EI is rendered meaningless [38]. They furthermore argue, that therefore the application of EI is better suited for niching applications where the goal is not to find one global optimum but rather to identify as many local basins as possible. Consequently, parallel model-based optimization requires a new adaptation that results in a less explorative search to quickly and efficiently converge to an optimum.

One inherent problem of developing such parallel modelbased algorithms is that even though they are meant to optimize expensive functions, they can usually not be benchmarked on such. Firstly, the high cost of each function evaluation renders extensive studies infeasible. Additionally, there are not many such problems openly available as they often lie under companies' confidentiality or require proprietary software to run. Daniels et al. published a suite of three CFD optimization problems meant for benchmarking [8]. While this is a good starting point, many more functions would be required. In the realm of cheap objective functions, large and well-documented function sets such as the Black-Box Optimization Benchmarking (BBOB) suite [15] are available. The noiseless single-objective BBOB suite consists of 24 test function classes, subdivided into five function groups. Each function class can be scaled in dimensionality. Furthermore, shifted, rotated, and stretched function instances can be drawn 
from each function class. The landscape and optima of each of the functions are known. Hansen et al. gave a detailed overview of the functions of this benchmarking suite [17]. A similar suite for expensive benchmark functions would be required for a rigorous benchmarking approach. Alternatively, methods for creating test problems that provide similar characteristics to those of the expensive functions are necessary.

These requirements motivate our first two research contributions:

C-1 We propose a framework on how to efficiently benchmark parallel model-based optimizers. The framework combines methods for emulating costly benchmark functions, as well as data-driven generation of test instances from real-world problems.

C-2 A novel parallel model-based optimizer is introduced. The performance of the algorithm is compared to other parallel algorithms using the introduced framework.

A common special case of expensive-to-evaluate functions are those where the evaluation cost stems from computational experiments. In such cases, parallelization can be applied to multiple levels of the optimization, and computational resources need to be balanced. Haftka et al. [14] separated between two distinct categories: coarse- and fine-grained parallelization. Fine-grained parallelization describes the parallel evaluation of the objective function, e.g., running a CFD simulation with multiple cores to speed up a single evaluation. Coarse-grained parallelization describes the act of proposing multiple candidate points per iteration with a given algorithm (e.g., the population size of an evolutionary algorithm). For model-based algorithms, the batch size defines the amount of coarse-grained parallelization. The batch size states how many points per iteration are selected on the surrogate model, to be then evaluated in parallel. We assume that adapting the batch size of an SBO algorithm can drastically change the behavior and performance of the algorithm. This is later shown experimentally.

In most application cases, a fixed amount of CPU cores is available for the optimization. Therefore, the usage of these cores has to be shared between coarse-, and fine-grained optimization. If more cores are assigned to each objective function evaluation, then the evaluation time per evaluation will be lower, yet, fewer proposal points of an algorithm can be evaluated in parallel. Assigning more or fewer cores to one of the levels changes the overall system's efficiency. A proper balance between the levels is crucial for performant optimization. Yet, tuning the batch size of an algorithm for a specific objective function is usually infeasible due to the high cost per evaluation.

We investigate approaches to automatically configure the batch size of parallel model-based algorithms by extracting specific properties of the functions landscape. Mersmann et al. introduced Exploratory Landscape Analysis (ELA) as a method to match optimization algorithms to problems efficiently, i.e., by extracting properties from problems with few evaluations [26]. The work defines eight high-level expert features to classify the BBOB test set. These features describe, e.g., the level of multi-modality, the global structure, the separability, and homogeneity in a rather subjective manner.
Mersmann et al. suggested a set of low-level features, e.g., convexity, curvature, local search, y-distribution, with a total of 50 numerical sub-features to characterize the problem structure [25]. One of the first successful applications of the promising combination of automated feature computation employing ELA and Machine Learning to address the algorithm selection problem can be found in [21]. They successfully train algorithm selection models on algorithm results on the BBOB test set.

Similar approaches can be applied to automatically determine a suitable batch size for SBO algorithms. The efficiency of specific batch size configurations is analyzed in an extensive benchmark study. The goal is to determine combinations of function landscapes and dimensionality that advocate for a certain batch size. This leads to our third contribution:

C-3 We investigate the possibility of predicting efficient batch size configurations for parallel SBO algorithms based on landscape features in a benchmark-based online algorithm configuration approach.

The online algorithm configuration measures certain landscape features at each iteration in order to predict the most effective batch size for the next iteration. The described approach is applied to predict algorithm configurations on unseen instances of BBOB test functions. Furthermore, the prediction is then applied to function instances generated by the data-driven approach to evaluate the quality of new functions. We aim at making all of our work publicly available and reproducible. This includes all program codes, figures, and experiment data.

The rest of this paper is structured as follows: After a brief overview of related work in this field, Section II introduces the new Batched Multi-Local Expected Improvement (MLEI) algorithm, as well as ideas on simulation-based test function generation. The implementation of these is discussed in Section III. Results are presented in Section IV and finally discussed in Section V.

\section{MethodS}

\section{A. Related Literature}

Investment Portfolio Improvement (IPI), originally introduced by Ursem [36] is a sequential strategy, creating sample points with different compromises between exploration and exploitation. This strategy can be adapted to create any required amount of sample points in parallel by generating multiple sample points, each optimizing a different priority compromise between exploration and exploitation.

Bischl et al. used the uncertainty measure of Kriging as an independent objective in multi-objective optimization, the other objective being the predicted value (PV) of the model (exploitation) [5]. Furthermore, they compared two distance measures which are optionally included as a third objective. This is done to increase the diversity of the selected points on the pareto-front. The implemented criteria are nearest neighbor and nearest better neighbor.

De Ath et al. [10] argued similarly to Wessing et al. [38] that more exploitation is beneficial to a sample efficient search with SBO. They adapted this initial work into a parallel 
sampling scheme coined the $\varepsilon$-shotgun [9]. Firstly, a single new point is sampled, either by optimizing the PV of the model or with a small chance $\varepsilon$ in a more explorative fashion. Then, locally around this single point, additional samples are drawn randomly. Hence the name $\varepsilon$-shotgun, representing the random-ish spread of shotgun fire to a single point. In theory, parallelization can be arbitrarily increased in this fashion, yet, a large batch size also means that a large number of points is randomly drawn around a single sample.

Rebolledo et al. [30] showed that parallelization can also be used in situations where the best choice of kernel, model, or infill criteria is unknown. A number of samples can be generated by building multiple differently configured models and optimizing them with different infill criteria in parallel. Each such combination creates a new sample per generation. The approach showed promising results, yet it is not easily scalable as a new implementation is required for each additional sample point.

\section{B. Batched Multi-local Expected Improvement}

In order to circumvent the downfalls of a too explorative parallel search (as described in Section I), we propose the Batched Multi-Local Expected Improvement (ML-EI) algorithm. ML-EI combines the explorative search of Q-EI with an exploitative search in the form of multiple parallel local optimizers. As Wessing et al. described [38], expected improvement is well suited to identify multiple interesting search basins with possible local optima. We make use of this by starting multiple parallel local searches in these basins. These searches aim to exploit local optima or possibly even the global optimum. Furthermore, an adaptive search scheme is used. At the beginning of a model-based optimization, search space exploration is required. Placing points in unknown regions of the search space can be useful for increasing the global model quality [32]. As the search progresses and less budget remains, exploitation becomes more important. Therefore, our search scheme traverses multiple phases. The goal is to gradually adapt from a very explorative search to finally a purely exploitative search based on the remaining budget. The structure of the ML-EI algorithm is shown in Figure 1. The budget-based phases are explained with an example batch size of four in Figure 2.

Starting in phase (1), an initial design for the optimization is created by Latin Hypercube Sampling [24]. The design of size $k$ is evaluated in parallel, based on the configured batch size, which in most cases will be smaller than $k$. The budget that remains after the initial design is split into batch size phases of equal budget, resulting in a total of $n=$ batch size + 1 phases. Initially, the goal of the optimization is to search very exploratively. Phase (2) only employs the Q-EI algorithm. Therefore, at each iteration batch size points are generated with the Q-EI criterion. To give an example, consider Figure 2: The algorithm is using a batch size of four. This means that there are a total of $n=4+1=5$ phases, and in each iteration of the algorithm, four points are generated. Throughout the phases, the amount of points that are generated per iteration does not change. Only the method with which these points are

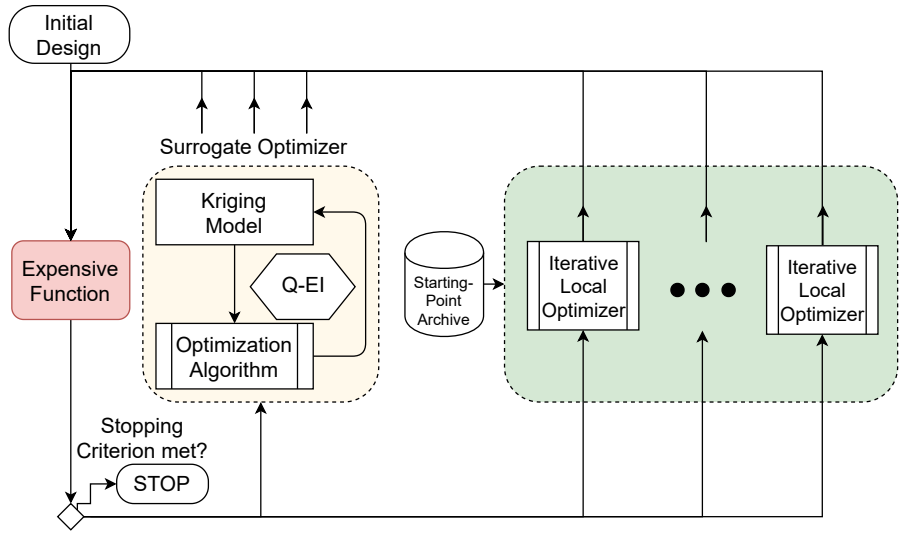

Fig. 1: ML-EI algorithm: points are generated in an initial design (white), as well as later by the surrogate optimizer (yellow) and multiple local searches (green). They are expensively evaluated on the objective function (red) until a stopping criterion is met. The starting point archive contains the initial design and all points proposed by the surrogate optimizer.

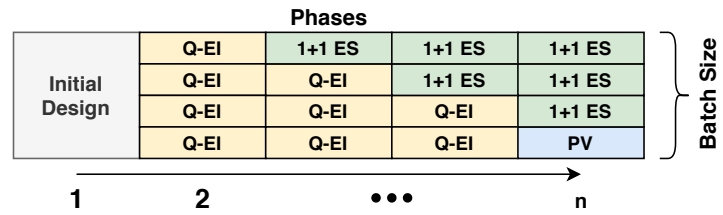

Fig. 2: Graphical example of the budget-based phases of the ML-EI algorithm for a batch size of four. After the evaluation of the initial design, the later stages represent how many points are generated with which method (Q-EI, 1+1 ES and PV) per iteration.

generated changes from more points invested into exploration to points being invested into exploitation.

In each following phase (3) .. (n), one fewer point is proposed with the Q-EI algorithm, and one local optimizer will be started. The local optimizers run in parallel to the modelbased algorithm. We employ a simple 1+1 evolution strategy (ES) as our local optimizer [31]. The 1+1 ES benefits us in this framework, as it only requires small budgets while still being able to improve from any starting point. Using the standard $1 / 5$ th rule for step size adaptation, it can quickly adapt to given search spaces to perform a simple local search. The 1+1 ES is just one possible choice of many and could theoretically be drop-in replaced with any other local optimizer. The only requirement for the local optimizer is to be efficient in lowbudget scenarios. It does not require, nor should it have any properties that would help with global optimization as its only task is to descend into the nearest basin.

The local optimizer directly evaluates the expensive function. It does not use any knowledge of the surrogate model, i.e., it does not use predictions of the model to guide the local search. The starting point of each ES stems from an archive that includes the initial design and is filled at every iteration with the newly evaluated Q-EI points. A new ES is started on the point in the archive with the best objective function 
value. If a point was already used to start another ES, it will be skipped, and the next best point is chosen. Points that the ESs propose are explicitly not included in this archive, even though they might include the best seen point. This is done to reduce the chances of two or more local searches running into the same basin.

Contrary to the ES, which is not using any information from the surrogate model or other ES evaluations, the surrogate model is fitted on all available data in each iteration. Thus, the model includes the initial design, all Q-EI points, and all ES evaluations. The predictive quality of the models benefits from each evaluation. Additionally, the many sample points that an ES places into one local area largely reduce the expected improvement for that same area. This significantly reduces the chances for the model to place additional samples into an area that is already taken care of by an ES.

In the last phase of the optimization batch size-1 ES instances are running in parallel, and the model proposes one additional evaluation point. Our choices are based on results and arguments presented in [32]. The budget spent on exploration to improve model quality becomes less and less beneficial, the less budget remains. Therefore, the last phase does not employ the single-core variant of EI anymore. Instead, the infill criterion is replaced with the predicted value of the surrogate model itself. Essentially creating another pure exploitation step, where only that point is evaluated, that the model believes to have the best objective function value, without any consideration of the model uncertainty.

\section{Benchmark Based Algorithm Configuration}

As an addition to the ML-EI algorithm, we propose methods for selecting suitable batch sizes for a given objective function. Facing an expensive-to-evaluate optimization problem, any existing knowledge about the problem landscape can help to configure an algorithm properly. Yet, often the only knowledge available can be gained by evaluating the problem itself. When such a problem has to be approached, parameter or algorithm choices can hardly be made. Given that in most cases at least the dimensionality and also the cost of the objective function are known, we consider a scenario where the choice was already made to use SBO to handle the high evaluation cost. However, even then, some necessity for algorithm configuration remains. Tuning the algorithm on the expensive problem is infeasible, as there is no budget for running multiple configurations observing which parameters work well. We consider two options to define a suitable batch size for parallel algorithms.

The first approach is based on an offline benchmarking study. The given algorithm can be benchmarked over a wide variety of cheap functions with different landscapes. It will be possible to observe which parameter settings work well for which kinds of problem landscapes. Furthermore, robust parameters that work across multiple landscapes, as well as 'niche' kind of parameters that work only in very specific setups, can be identified. Running these benchmarks on cheapto-evaluate functions is likely less costly / computationally intensive than a single or just a few evaluations of the expensive problem. The gained knowledge can, for example, be applied to the algorithm by choosing a robust setting for the unknown landscape of the expensive problem.

The second approach aims to change the algorithm configuration during the optimization runtime. This approach still involves the offline benchmarking phase. Yet, the results of these benchmarks are then used to fit a prediction model. The ELA features of each of the benchmarked functions can be measured, as well as which algorithm configuration worked well for which function. The task of the model is then to accept such a set of landscape features of a new problem and predict a well-working algorithm configuration based on that. If such a model could make correct predictions during the algorithm runtime, then algorithms would always be correctly configured even for new and unseen problems. This assumption relies on the correct prediction of the model, which will be hard to achieve.

In the following, we will briefly introduce the implementation of the proposed approach: To calculate the landscape features of given functions, all evaluated points are recorded. We use the R-package flacco [22] to generate features based on these sample points. In flacco itself, the measurable features are arranged in sets. We exclude all feature sets that require additional function evaluations, as we do not want to spend any budget on the feature measurements but rather only reuse the already available evaluations. Furthermore, the distribution features ela_distr were excluded. This feature set often yielded numerical problems when it was calculated on the ES sample points, which are locally close to each other. The final prediction model uses the following feature sets as input parameters: problem dimensionality (ela_basic), meta model features (ela_meta) and information content features (ela_ic).

Since the model is supposed to predict which algorithm configuration works well on which kind of function, the algorithms performance is measured on each available test function and with each available configuration. The winning configuration is determined to be the algorithm configuration that delivers the best median objective function value on a given problem. Dependent on the optimization demands, the median might also be replaced with other measures, for example, the worst observed result if looking for a robust approach. The exact details on these benchmarks and the used test functions will be covered in Section III.

The desribed landscape features $\rightarrow$ best algorithm configuration data can be used to fit a prediction model. As we aim to show a proof of concept, we choose a rather simple random forest [6] model for its robust properties. Replacing the model in this framework is easily possible. The fitted model can be incorporated into the optimization loop of any parallel optimizer to suggest the batch size for upcoming iterations. We exemplify this procedure by adding the batch size prediction to the ML-EI algorithm that was introduced in Section II-B. Each time new sample points are expensively evaluated on the objective function, flacco is used to estimate the characteristics of the given objective function. The measured features are fed into the random forest model, which was trained on the benchmark data. Based on the features, it will give an estimate of which algorithm configuration should work best for the 
problem at hand. Therefore, before the start of each new iteration of the ML-EI algorithm, the algorithm is configured with a new batch size. At each iteration, the number of proposed points that shall be evaluated in parallel can change based on the current assumptions of the optimized problem landscape.

\section{Simulation-based Benchmarks}

Benchmarking algorithms on real-world functions is often constrained by the limited availability of such functions. Test function generation by simulation tries to alleviate this problem. Previous work describes a method for the generation of continuous optimization benchmark functions via the simulation of Kriging models [39], [40]. Details of Kriging prediction and simulation are, e.g., provided by Cressie [7]. Further details on how Kriging prediction is usually used in expensive optimization are, e.g., given by Forrester et al. [12].

The core idea of Continuous Optimization Benchmarks by Simulation (COBBS) is to generate new problem instances from existing data, especially in contexts where 'real' problem instances (i.e., the corresponding objective functions) are unavailable or too expensive for an in-detail experimental investigation such as a benchmarking study [40].

In this work, we will add an extension to the Kriging simulation in COBBS. The Kriging model used by Zaefferer and Rehbach [40] is a stationary one, with a constant trend. This will often limit the quality of the model, and hence, the quality of the produced test instances. The single stationary model will either be able to deliver a model that fits well to the global features of the input data or excel in reproducing the finer, local problem structures. As only one activity parameter is fitted per problem dimension, it is hard to represent the data's local and global features.

We use a two-stage approach to address this issue, which is to some extent similar to a single round of boosting [19]. When training the Kriging model, a first-stage model is generated with the nugget effect enabled. The nugget effect allows the Kriging model to regress and smoothen the data [12]. This enables to fit the global trend of the data but will ignore (i.e., smoothen) finer structures of a landscape.

Once this model is trained, a secondary Kriging model is fitted to the first-stage model's residuals. The second-stage model does not employ the nugget effect and will attempt to interpolate, i.e., fit the training data exactly. Thus, the secondstage model can account for any local structures that are not well represented by the first-stage model. The combination of both models (i.e., the addition of the predictions or simulations from each model) produces a model with a flexible, nonstationary trend. Importantly, the non-stationary trend itself can be varied for the simulation.

To further benefit from the two-stage structure of our model, we also adapt how the activity parameters $\theta_{i}$ are determined for the second-stage model. The parameters of the first-stage models are determined via numerical optimization of the likelihood as usual, with relatively generous lower and upper bounds for the search (here: lower bound $10^{-6}$, upper bound $10^{12}$ ). These bounds are identical for all dimensions $i$.
We aim to build a first-stage model which fits slower changes in the data (i.e., the global trend). Hence, we expect that the activity parameters $\theta$ of the Gaussian kernel that we use, i.e., $\mathrm{k}\left(\mathbf{x}, \mathbf{x}^{\prime}\right)=\exp \left(\sum_{i=1}^{n}-\theta_{i}\left|x_{i}-x_{i}^{\prime}\right|^{2}\right)$, are much smaller (i.e., correlations decay more slowly) than in the second-stage model. To exploit that assumption, we specify different lower bounds for the second-stage model: for each dimension $i$, the respective lower bound of $\theta_{i}$ in the second-stage model is the respective $\theta_{i}$ determined when fitting the first-stage model. This reduces the size of the likelihood search space and hence simplifies the search.

\section{EXPERIMENTS}

\section{A. Codes and Reproducability}

In order to make all described experiments reproducible, all codes of all experiments are published: https://doi.org/10.5281/zenodo.5235485 . Additionally, the repository hosts all recorded raw data of the experiments and all sets of detailed result plots for each experiment. This also makes it possible to run future algorithm comparisons in the same manner, reusing the already existing results without spending additional computational resources.

\section{B. Benchmarking Fixed batch sizes}

The first set of executed experiments concerns a general algorithm comparison where the batch size of the algorithms is predefined and constant. In this scenario, a fair comparison of an algorithm's performance can only be drawn when comparing it to other algorithms using the same batch size. When these comparisons are drawn, an objective performance measurement is required.

A common strategy to judge an algorithm's performance on expensive problems is to compare each algorithm's best found objective function value, given a certain amount of function evaluations. For parallel algorithms, this performance criterion has to be changed as they can evaluate multiple candidate solutions per iteration. Therefore, the first set of experiments is judged on the results achieved after a fixed amount of iterations by each algorithm. This makes algorithms with the same batch size directly comparable, as they will also have used the same amount of total function evaluations. A comparison between the results of different batch sizes is covered in the following Section III-C.

While the benchmarked algorithms aim to solve expensive to evaluate objective functions, cheap to evaluate functions are necessary to enable in-depth benchmarks without requiring large computational budgets. Therefore, we employ a commonly used benchmark suite: the Black-Box Optimization Benchmarking (BBOB) suite [15]. The expensiveness of the benchmark functions is emulated by limiting the number of objective function evaluations for each optimizer. All experiments are implemented with a recent GitHub version of the benchmarking suite (v2.3.1).

To judge the performance of the model-based algorithms, we first benchmark three baseline algorithms. Firstly, a uniform sampling random search as a lower baseline to performance. Next, we chose the standard python CMAES implementation by Hansen [16] as a well-known efficient optimizer 
on the BBOB function set. The CMAES uses a population of candidates at each iteration. It can, therefore, efficiently make use of multiple objective function evaluations in parallel. The population size of the CMAES was set to $4 \times$ batch size. Since ML-EI uses multiple 1+1 ESs for local optimization, the performance of the same 1+1 ES algorithm alone should be taken as the third baseline. Yet, a 1+1 ES only proposes one new candidate solution per iteration. To judge its performance compared to parallel algorithms, as many ESs are started in parallel as the batch size allows. The 1+1 ES implementation is based on the 'optimES' implementation of the R-package SPOT[3].

Both the CMAES and the 1+1 ESs are started with initial random uniformly sampled starting points. Each algorithm is given 50 iterations and is run with batch sizes $2,4,8$, and 16 , resulting in 100 to 800 objective function evaluations per run. The algorithms are run on all 24 BBOB functions, on two- and five-dimensional problems. For statistical analysis, 15 repeats are done per run. Each repetition is evaluated on a new instance of the given function class. However, each optimization algorithm sees the same 15 instances to make the comparison as fair as possible.

Next, multiple parallel model-based algorithms are benchmarked. We compare Q-EI, MOI-MBO, IPI, and our proposed ML-EI. Each model-based algorithm is initiated with a latin hypercube sampled initial design [24]. The design size for each algorithm is $2 \times$ problemDimension $\times$ batch size. Thus, the first $2 \times$ problemDimension iterations of each algorithm are used for the initial design.

Q-EI is implemented in R through the DiceKriging package, which is based on Ginsbourger et al. [13], [34]. The package method $q E I$ accepts multiple candidate solutions and returns their combined expected improvement. For the optimization of this infill criterion we employ the differential evolution [27] implementation of the package 'SPOT' [1], [2] (optimDE). This internal optimizer is given $1000 \times \log ($ problemDimension $)$ evaluations of the Q-EI criterion.

The IPI criterion is implemented based on [36]. Internally, we use the same SPOT Kriging model and optimDE with the same budgets as for Q-EI.

The implementation for MOI-MBO is taken directly from the authors' $\mathrm{R}$ implementation: 'mlrMBO' [4]. More specifically, the package is configured to use the Multi Objective Infill - Model-Based Optimization (MOI-MBO) setup that performed best in their original experiments. The configurations are reported in [5]. According to their best-performing configuration, we implemented ID 10 for our experiments. Apart from that, the same initial design sizes and internal optimizer budgets as for IPI and Q-EI are assigned to MOIMBO.

\section{Benchmarking for Multiple batch sizes}

In many optimization tasks, like, for example, the optimization of CFD simulations, the objective function itself can be parallelized (fine-grained parallelization). In a system with a fixed amount of CPU cores, the time per evaluation and the batch size (coarse-grained parallelization) of an algorithm are

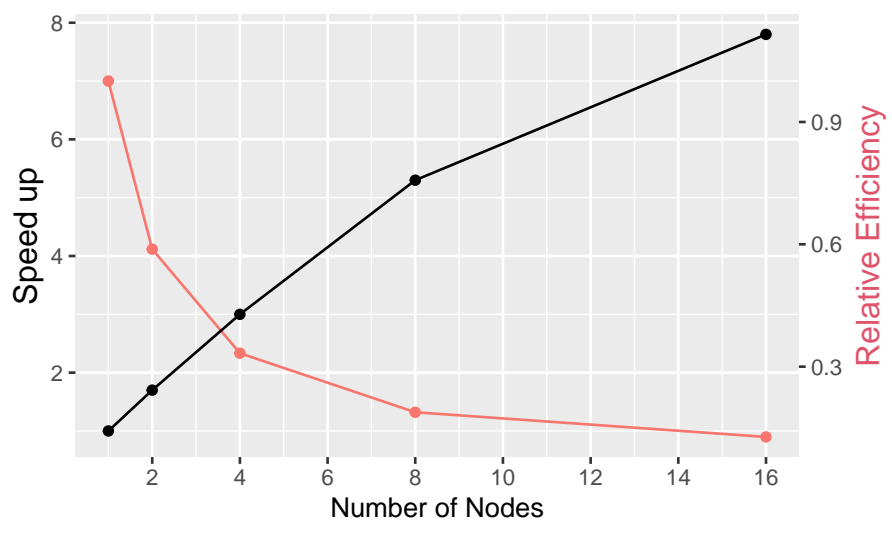

Fig. 3: Example decrease in measured speedup vs. parallel efficiency for an increasing number of computation nodes. Numbers are taken from example measurements of a small CFD simulation and will largely differ for other problems. Figure idea based on [37].

linked together. Given a total amount of cpu cores in a system $\left(n_{T}\right)$ and an amount of cpu cores per fine-grained evaluation $\left(n_{f}\right)$ the following equation must hold true:

$$
n_{T} \geq n_{f} \times \text { batchsize }
$$

If each evaluation is assigned with more cores, then the evaluation time will be lower. However, at the same time, not as many evaluations can be run in parallel, reducing the batch size of the optimizer. Therefore, the efficiency of an optimization is not only dependent on the optimizer itself. Instead, the corresponding best batch size for an algorithm needs to be determined, taking into account the evaluation runtime at a given batch size. This is shown with an example measurement in Figure 3.

The benchmarks described in the previous section allow for a static comparison of multiple algorithms of the same batch size. Yet, they do not allow for a fair comparison of algorithms of different batch sizes. Considering Figure 3, algorithms with different batch sizes do more or fewer evaluations in the same time, which also results in different amounts of wall-clock time spent per iteration.

If no additional cost was associated with a rising batch size, then usually the highest possible batch size should be chosen, as simply more evaluations are possible. Yet, as previously described, if only a certain amount of computational resources are available (which is almost always the case), then increasing the batch size comes at the cost of a lowered fine-grained parallelization. Therefore, benchmarks that try to compare algorithms of different batch sizes need a measure that incorporates this indirect cost.

Additionally, we seek a method that allows the transfer of the 'cost' of an expensive function to cheap functions. We employ a two-step approach to fairly judge the parallel efficiency of an optimizer together with a specific objective function. Step one requires a runtime measurement of the original expensive function. These measurements can be collected by using a few samples, measuring the evaluation runtime of 
the problem, for example, a simulation. Each simulation is run with a different number of cores to observe the runtime scaling as reported in Figure 3. Based on these evaluations, it is possible to estimate a function $f_{t}\left(n_{f}\right)$ describing the amount of time needed per evaluation, given a certain amount of cores $n_{f}$. The trial simulations used for the measurements can also already be part of the initial design. In that case, they would not require any additional budget taken away from the optimization run itself.

To measure the efficiency of all possible trade-offs between coarse-, and fine-grained parallelization, essentially all possible amounts of CPU cores per objective function evaluation could be recorded. However, often a lot of combinations are much less efficient and can already be ruled out by process knowledge.

To give just one example: If the total amount of available CPU cores in a system is not a multiple of the number of cores used per simulation, then some cores are left unused, and thus the overall efficiency drops.

For the sake of simplicity, we only use powers of two $(2,4,8,16$ cores $)$ in our experiments. If the runtime between repeats of the same evaluation varies slightly, then the measurements might be reported as the mean runtime of the repeats. However, this approach is not meant to cope with large runtime deviations in repeated function evaluations with the same amount of CPU cores. If the evaluation time largely varies, an asynchronous evaluation scheme such as the one presented by Kotthaus et al. [23] is likely better suited.

Knowing the execution times $f_{t}\left(n_{f}\right)$ of an expensive objective function allows for step two: scaling the measured costs and applying them to cheap functions. The empirically determined runtimes can be translated into 'costs' for cheap to evaluate functions like those of the BBOB function set. For this purpose, we first again have to consider the link between the batch size and the number of CPU cores used per evaluation.

For example, let's assume that we have a system with 32 cores for our optimization. If we were to choose a batch size of eight, then this would directly translate into $32 / 8=4$ cores to be used per evaluation. Whereas a lower batch size of, let's say four, would lead to $32 / 4=8$ cores that can be used per evaluation. Thus, a lower batch size is associated with a lower wall-clock cost since more resources can be used to speed up a single evaluation.

Finally, the translation from an execution time $\left(f_{t}\right)$ to an evaluation cost $\left(f_{c}\right)$ given a certain batch size $(b)$ is described in Equation 1. First the execution time with an amount of cores $n_{f}=\frac{n_{T}}{b}$ is observed. This time is then scaled with the base evaluation time, that an evaluation with a single core would take $\left(f_{t}(1)\right)$.

$$
f_{c}(b)=\frac{f_{t}\left(\frac{n_{T}}{b}\right)}{f_{t}(1)}
$$

To give just one example of this scaling procedure, consider the following scenario: Assume that an objective function evaluation with a single core took five hours of computation time. Given a total of 32 cores in the system, the algorithm could be configured with a batch size of 32 and would use exactly one unit of budget per iteration. Yet, following the graph of Figure 3, if we were to use four cores per evaluation, we would gain a factor 3.5 evaluation speedup. The objective function evaluation would only require roughly 1.4 hours and therefore only 0.28 "units of budget" per iteration. Yet, we would not be able to evaluate 32 points in parallel, but instead only 8.

In summary, in the proposed framework, algorithms are not given an iteration budget any longer, but instead a budget relative to a base evaluation time. This allows for easy back and forth calculations to estimate real-time performance, even for cheap to evaluate functions. Furthermore, it allows plotting the performance of multiple algorithms with different batch sizes on a unified axis, comparing their estimated total runtimes. This method is used to compare the previously described algorithm benchmarks across batch sizes. Results will be shown in Section IV.

\section{Benchmarking the Adaptive Algorithm}

1) Benchmarking on $B B O B$ : The final section of experiments regards the batch size modeling and online prediction for the ML-EI algorithm. In order to predict the performance of algorithm configurations on specific functions, the results of the ML-EI runs with static batch sizes were analyzed. To create a simple proof-of-concept, we only compare two configurations: low (batch size of 2) and high (batch size of 8). The results on each BBOB function and each dimensionality are treated separately. The batch size with the lowest median across 15 repeats on such a function-dimension pair is reported as the best performing configuration for that pair. The ELA features are measured separately for every function instance. Thus, the collected training data consists of 24 functions $\times$ 2 dimensions $\times 15$ instances $=720$ samples. Each sample represents one pair of landscape features together with a recorded best batch size. The random forest prediction model is built using these 720 samples.

The prediction model is then used in additional optimization runs, where it continuously changes the algorithms batch size. For this, at each iteration, the ELA features of the function instance at hand are calculated and used for prediction. This means that at the beginning of an optimization, these predictions are only based on very few sampled points. The longer the optimization is running, the more samples are available and the better the ELA feature estimate, as well as the model prediction, should get.

For the first set of the online adaptation experiments, the static algorithms were run on the BBOB instances 1-15 of each function to generate the training data. Then, the model was tasked to predict during the optimization on instances 1630. Thus, we employ a similar train/test split as Mersmann et al. [25].

Therefore, if the model is capable of correctly predicting the batch size for those instances, it can be argued that the model can accurately generalize to new unseen function instances. However, remember that the ultimate goal of such an online adaptation would be to correctly predict the batch size of a function class that was never seen before. If this goal could be met, then such an approach could be perfectly applied 
to costly real-world problems where no a priori knowledge is available. As the $\mathrm{BBOB}$ instances only represent changes through translation and stretching, the resulting instances still have high similarities. Thus, making it easier for the described model to correctly classify which function class is represented and therefore which algorithm configuration should be chosen.

2) Benchmarking on Simulated Functions: To circumvent the described problem, the predictive power of the adaptation approach shall be tested on a more diverse set of new objective functions. To create more independent test functions, we employ Kriging simulations with the methodology as described in Section II-D. One test function is created for each of the 24 BBOB function classes in their two- and five-dimensional forms. The simulations are fitted by training the Kriging model with 400 sample points each. The samples stem from the previous optimization runs on those functions. We use the same approach of generating training data as described in [40] because the samples a) cover the whole search space in a reasonable manner and b) cover potentially optimal areas more densely, thus being able to represent both local and global patterns in the data.

The respective results were taken from previous runs of the static ML-EI algorithm with a configured batch size of eight. The models are trained with nugget effect for the first level model to account for a rough global trend (see Section II-D). The lower and upper bounds on the kernel parameters $\theta$ are $10^{-6}$ and $10^{12}$ respectively, accounting for a wide range of activity in the search landscapes. Based on the fitted models, the simulations are composed of $100 \times$ dimension superimposed cosine functions. We perform non-conditional simulations, i.e., the simulation will only reproduce the estimated covariances but not the actual training data.

The adaptive algorithm is then run on all simulated functions trying to predict the best batch size configuration. The prediction model is still only fitted to the data observed on BBOB functions. In order to measure the true best batch size, the static ML-EI algorithm is also applied to each function. Both algorithms are again run for a total of 15 repeats.

\section{RESUlts AND Discussion}

\section{A. Static batch size Experiments}

The first set of results is reported for static batch sizes. We compare the results of each algorithm given a certain fixed batch size. Figure 4 shows a set of convergence plots on different BBOB functions. The specific plots were handpicked as they represent interesting features that will be discussed in the following. A detailed set of all convergence plots, as well as boxplots for each optimization run covering statistical results, are parts of the supplementary material.

The left plot in Figure 4 shows results on the sphere function with a batch size of two. This result best represents the desired behavior of the ML-EI algorithm. At the beginning of the optimization, ML-EI behaves exactly like Q-EI. Thus, they also show the exact same performance for the first part of the optimization. Yet, after switching to the exploitative search step, quick progress is made. The combination of the $1+1 \mathrm{ES}$ and the PV criterion works well on the simple landscape of the sphere function, quickly outperforming the other algorithms. It is also worth noting that after good initial progress, the modelbased methods all stagnate in their search. No further progress is made, although the problem at hand should be easy to solve. This is likely due to the fact that parallel infill criteria largely rely on Krigings uncertainty estimate and avoid placing points in already sampled regions. While this aids the exploration of the search space at the beginning of an optimization, it renders local exploitation impossible.

The second plot from Figure 4 shows results from the ellipsoid function. While the ellipsoid function still features a smooth, unimodal landscape, its key distinction point is the high conditioning of the problem landscape. It was picked for discussion here, as high conditioning (which is present in many BBOB functions) was the strongest differentiator in algorithm performance throughout the benchmarks. Due to the high conditioning, results with high function values of up to $10^{6}$ are to be expected. All algorithms deliver considerably worse results compared to the results on the sphere function. The CMAES is much closer to the model-based methods in this comparison. Yet, the largest difference in results can be observed for the ML-EI algorithm. The previously observed quick progression of the exploitation phase is largely missing on the ellipsoid function. This is likely because the $1+1 \mathrm{ES}$ stepsize adaptation does not work well on highly conditioned functions. The MOI-MBO works best in this scenario, significantly outperforming the other algorithms.

The third set of results in Figure 4 was picked to represent a common behavior for ML-EI on multi-modal functions. Similar behavior was observed on other functions as well but has shown up in the clearest form on the Rastrigin function. Firstly, it is clear to see that the pure 1+1 ES does not cope well with the many local optima, even being outperformed by random search. Yet, the combination of Q-EI and the same local search in the ML-EI algorithm delivers continuous progression on the search space. Thus it can be argued that the ES strongly synergizes with the explorative global search of Q-EI.

The next set of results is aggregated in a dense format into Figure 5a and Figure 6. Both Figures show the results of all algorithms on all functions and dimensionalities. Figure 5a shows the processed results of a statistical analysis.

As we cannot expect our data to follow a normal distribution, we decided to apply non-parametric tests as suggested by Derrac et al. [11], as these make fewer assumptions about the underlying data. We apply the base-R package 'stats' to implement the Wilcoxon test, also known as Mann-Whitney test [18]. Results are accepted as statistically significant if pvalues smaller than 0.05 are reported.

For each combination of function and dimension, the colored boxes in Figure 5a represent the set of best algorithms for that combination. If only one algorithm is marked (colored different than gray) for a certain combination, then it outperformed all other algorithms with statistical significance. If multiple algorithms are marked, this indicates that there was no statistically significant difference between them. Starting off, it is noticeable that the harder a function or optimization task is, the less difference there is between algorithms competing 

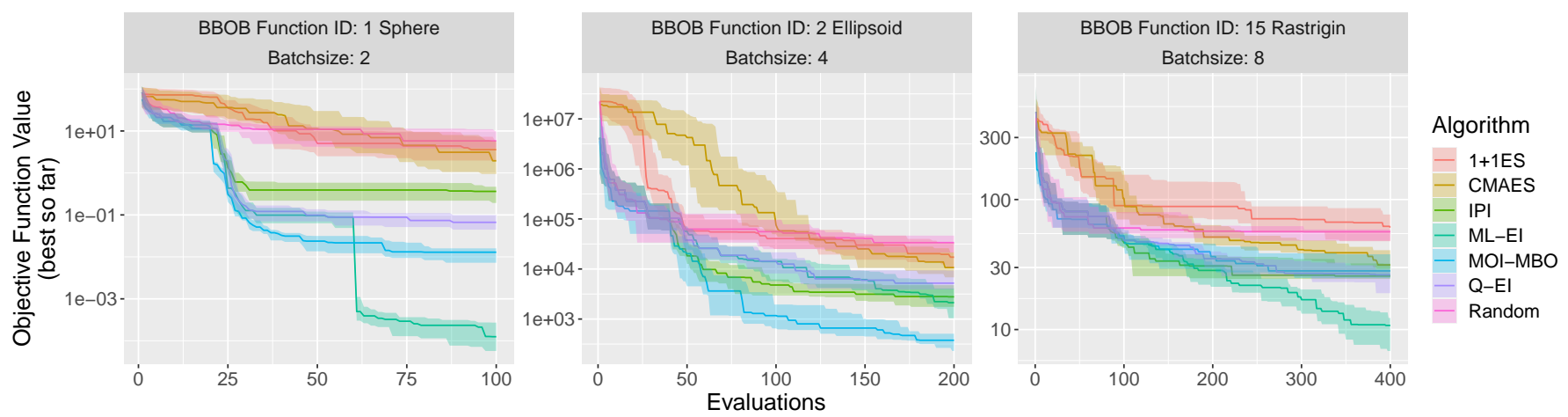

Fig. 4: Convergence plots showing optimization performance over algorithm runtime. The Y-axis shows the best so far found objective function value, the $\mathrm{X}$-axis the current evaluation. All plots show experiments on 5-dimensional functions. The solid line in the plot represents the median of all repeats, the transparent colored areas represent the upper and lower quartiles. The convergence behavior shown in plots is explained in detail in Section IV.
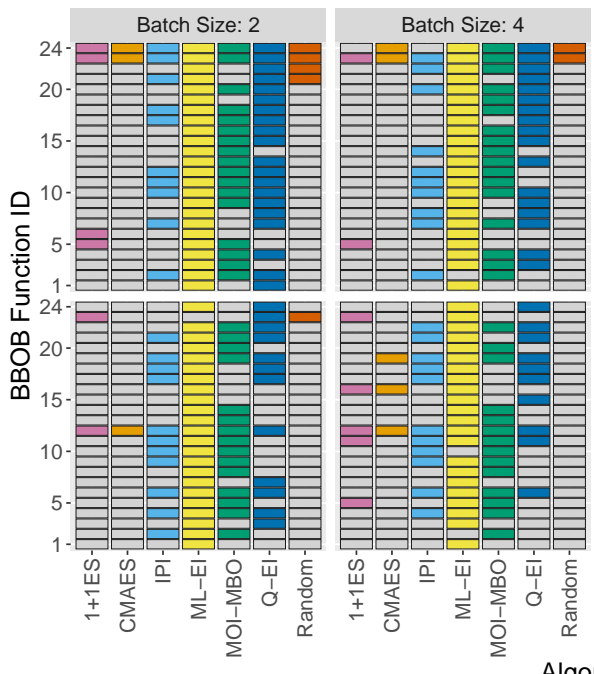

Algorithm
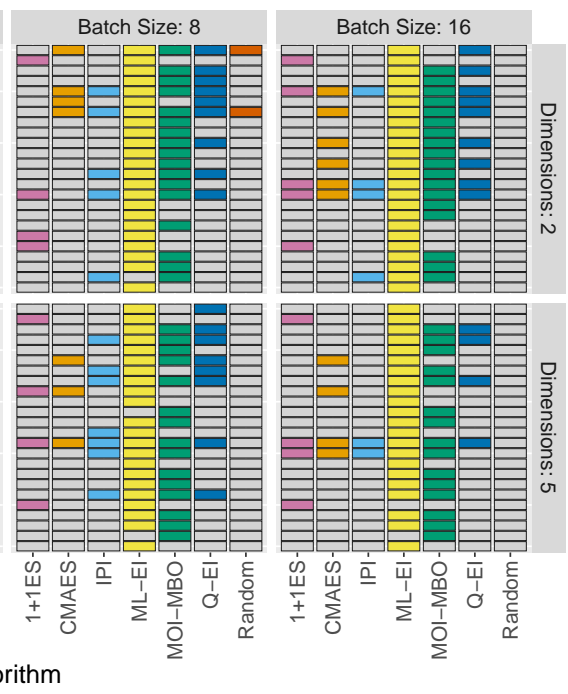

(a) Summarized statistical analysis over all algorithms and functions after using their complete budget $(100 \times$ batch size). If a box is gray, this means that the specific algorithm was outperformed with statistical significance. Colored boxes show that the algorithm is NOT outperformed in that setup. For example, top left corner of the plot: batch size 2, dimension 2, function 24 . No algorithm was able to outperform another one with statistical significance. Hence all fields are colored.

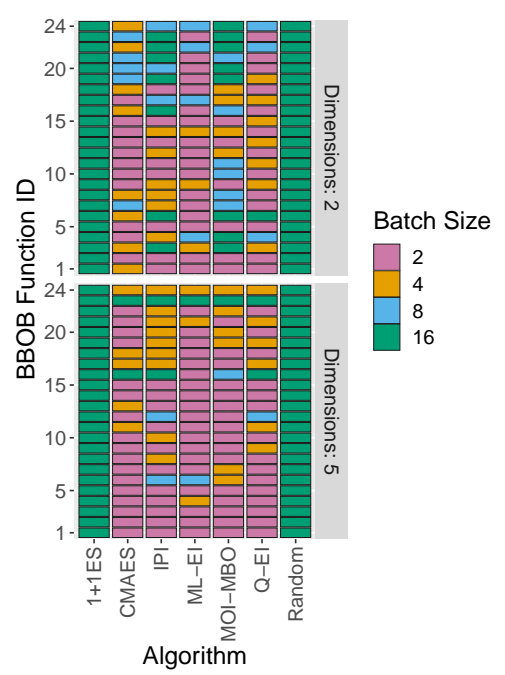

(b) Aggregated results showing the best median batch size for a given algorithm on a given function. Results taken from an examplary point in optimization time after ten time units.

Fig. 5: Aggregated results of the static batch size experiments. On the left, results for each batch size are shown. The right plot further aggregates and shows the best batch sizes.

on the functions. In the two-dimensional case with a batch size of two, on functions 23 (Katsuura) and 24 (Lunacek bi-Rastrigin), no algorithm outperforms any other algorithm. Furthermore, the random search is not outperformed on functions 21 (Gallagher's Gaussian 101-me Peaks) and 22 (Gallagher's Gaussian 21-hi Peaks), even outperforming the 1+1 ES, CMAES, IPI, and MOI-MBO. The hard combination of a low evaluation budget (due to the batch size of two) and the weak global structures and high modality of the function leads to poor performance of all applied algorithms. With increasing problem dimension and batch size, the random search baseline is less often on par with the other optimizers. With a batch size of 16 , random search is always outperformed.

Interestingly, the parallel 1+1 ES can not compete on the sphere function. This is arguably due to the fact that the parallel restarts are not helpful on the unimodal sphere function, thus wasting evaluations. The CMAES performs increasingly well with higher evaluation budgets. While it is only rarely able to compete in a scenario with a batch size of two, it can compete on a total of ten function/dimension combinations with a batch size of 16 . This coincides with other experiments that have shown that a CMAES starts outperforming modelbased methods when it is given enough budget [30]. This observation is further signified considering Figure 6. While the CMAES nearly never outperforms on any function for the lower batch sizes (once with a batch size of four on the Bent Cigar function, ID 12), it has the best median performance in five cases for a batch size of 16 . 


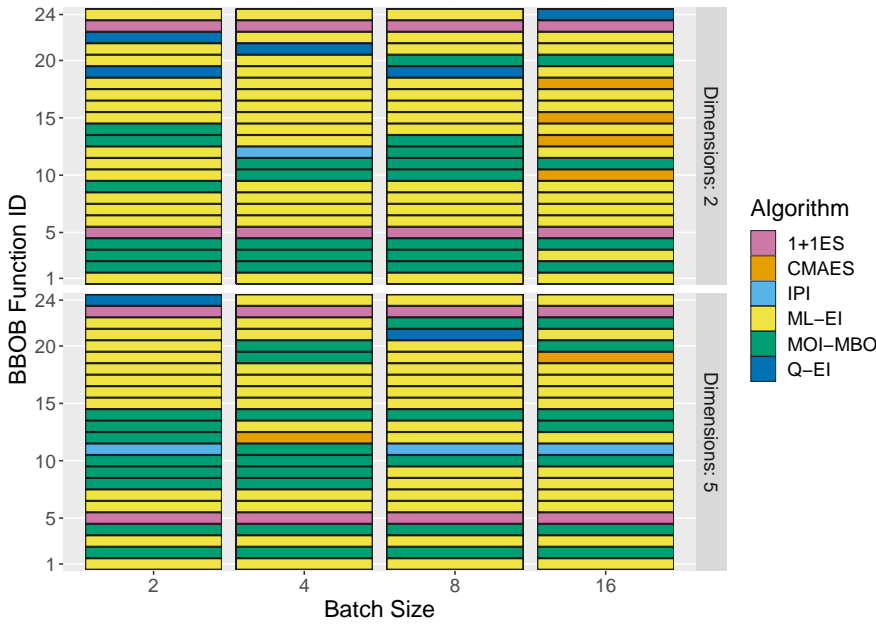

Fig. 6: Best performing algorithm on a given function. Each cell of the plot represents the repeats of multiple algorithms on a function (Y-axis), batch size (X-axis), and dimensionality (vertical split). The color indicates the algorithm with the best median objective function value after the optimization budget is depleted.

Overall, the model-based algorithms perform relatively well across most setups. Both IPI and Q-EI seem to do better for batch sizes two and four and are often outperformed on batch sizes eight and 16. This seems to validate the previously made argument, that these algorithms tend to do too much exploration with an increasing batch size. More exploitation is required to refine the results given the small number of iterations that the algorithms can do. MOI-MBO and ML-EI deliver more robust results across the board. On the five-dimensional cases, MOI-MBO is often outperformed on functions 15-18, which exactly coincides with the group of BBOB functions with adequate global structure. Furthermore, MOI-MBO is outperformed on some functions with weak global structure.

ML-EI delivers good and especially robust results. For almost all function classes, it resides in the set of topperforming algorithms, making it well applicable in a scenario where the function landscape is previously unknown. It is outperformed on functions with high conditioning like the two ellipsoid functions (BBOB ID 2 and 10). Considering Figure 6 , a broader look on the results can be taken, showing which algorithm actually performed the best on which function. Yet, here the best-performing algorithm is directly chosen by best median performance and thus does not necessitate a statistically significant outperformance. Still, this view can give interesting insights into which algorithms performs well under which circumstances. While ML-EI was nearly always in the set of well performing algorithms in Figure 5a, Figure 6 shows that it is often not the single best performing algorithm. In many cases MOI-MBO delivered the best median performance. In some cases ML-EI is outperformed by the CMAES, and in rare occasions even by Q-EI.

Figure $5 \mathrm{~b}$ analyses the algorithms in the newly introduced format of unit time. The results shown are not meant to actually judge these algorithms, as they only represent one moment in time for one specific set of costs of a computer simulation. Yet, we want to use them to present how the introduced benchmarking framework, that assigns costs in unit-time, can be applied, and what knowledge can be gained from the results.

Firstly it is easy to notice, that for both the $1+1 \mathrm{ES}$ and the random search, batch size 16 is always the best. With a batch size of 16 few iterations can be made, yet the total amount of evaluations is the highest. The random search does not lose efficiency when scaled/parallelized. The expected final result should only depend on the number of evaluations done, not on the number of iterations, which is correctly represented in the plot. For the 1+1 ES, this behavior is more interesting. As the plot was generated for a rather early point in optimization time, the step-size adaptation of the 1+1 ES did not have enough time to adjust to the different function landscapes. Thus, until that point, having more starting points seems more beneficial than having more iterations of local search. For the other algorithms, a clear difference is visible. The CMA-ES seems to prefer smaller population sizes with more iterations on most function classes. The same goes for the model-based algorithms as they report the most progress especially in the early iterations of their optimization. Having a few more iterations in such an early stage of the optimization helps on most functions. On problem 23 (Katsuura), where most algorithms were not able to outperform random search, batch size 16 performs best for nearly all algorithms, again indicating that a rather random search strategy with more sample points wins.

\section{B. Adaptive batch size}

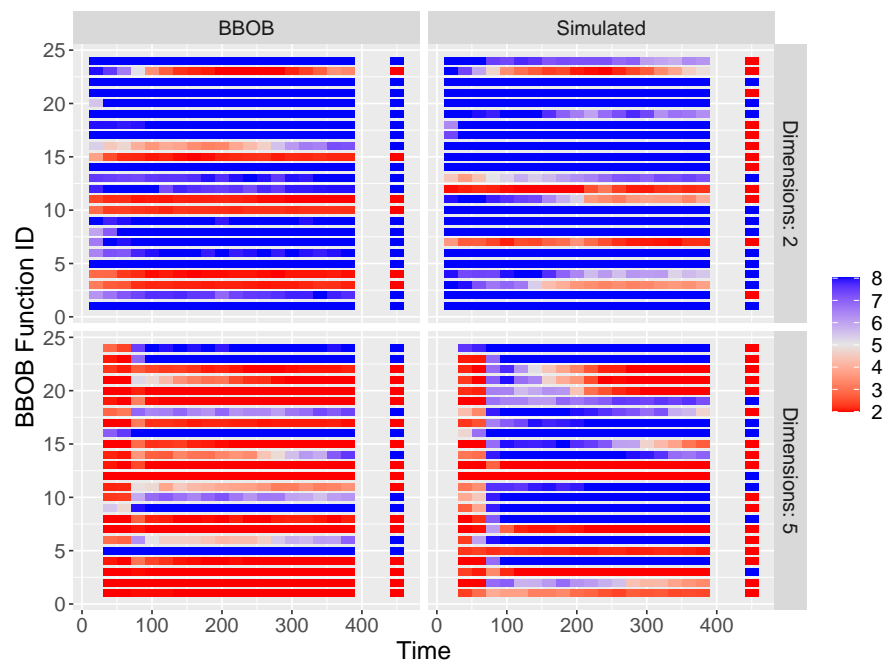

Fig. 7: Batch size predictions during adaptive ML-EI run. Y-axis: BBOB function 1-24. X-axis: optimization runtime scaled in base evaluations. The model only predicts batch sizes two or eight. The color of a cell shows the predicted batch size averaged over multiple repeats. The separated colors on the right of each plot represent the measured ground-truth of the static ML-EI variant that should be predicted. 
1) Results on $B B O B$ : The last set of results regards the predictive quality during the adaptive batch size optimization. The goal here was not to observe performance compared to other algorithms, but rather to analyze if the best-performing batch size for a given algorithm can be correctly predicted based on landscape features. Figure 7 shows the batch size predictions in summarized manner. Let's first consider the left-hand side of the plot, representing the results on BBOB functions. The plot is again separated into the 24 BBOB function IDs (y-Axis) as well as by dimensionality (two and five). The single separated column of blue and red rectangles shows the ground-truth that should be predicted. It was determined via experiments run with the static batch size version of ML-EI as described in Section III-D.

The left-hand side of the plot shows the predictions of the ML-EI runs with adaptive batch size. The color of each rectangle is averaged over the 15 repeats done on distinct BBOB function instances. Thus, a strongly colored red or blue rectangle represents that the model delivered the same predictions across all instances, whereas a white rectangle represents an iteration where different predictions were made across instances.

Notably, the plot shows that for most two-dimensional functions a high batch size performs better than a low batch size. The opposite is the case for the five-dimensional functions. At first, this seemed counter-intuitive. One might expect that more complex higher-dimensional problems might require a higher batch size for a more explorative search. However, also the experiments conducted by Rehbach et al. [32] indicated that exploitation is increasingly important for functions with higher dimensionality.

Overall, the results on the BBOB functions look promising. In most cases, the model correctly predicts the best batch size, even early in the optimization where only a few samples are available to calculate the ELA features from. In the final iteration the model correctly predicts all 24 function classes, both on the two- as well as on the five-dimensional problems. However, in seven out of the 48 cases ( 24 functions $\times 2$ dimensions), the correct prediction is not made for all 15 instances.

In these seven cases, we can also observe the highest variation in the predictions. Consider for example the fivedimensional version of function 14 (different powers function). For roughly three-quarters of the optimization runtime, a small batch size was predicted for most instances. Only in the final evaluations, the model was able to correct this. This might be due to the fact, that the landscape of the different powers function is very similar to other functions until the singular spike in objective function value is found.

Based on the results that were observed on the BBOB functions, we can argue that even a fairly simple model, e.g. our random forest setup, can easily predict a well-performing algorithm configuration for new instances of BBOB functions. It is surprising to see how few samples are necessary to calculate a set of ELA features that can be used for a correct prediction. Yet, these results only represent the ability to give predictions for instantiated functions of BBOB classes. As previously discussed, these can not be argued to be wholy new and unseen functions.

2) Results on Simulated Functions: The right-hand-side of Figure 7 shows the results on the test functions generated via Kriging Simulation. Again, the single separated column of colored rectangles represents the ground-truth that should be predicted by the model. The ground-truth is measured by running the static version of the ML-EI model on the newly generated functions and recording the best median performing configuration. As expected, the predictions on the simulated functions are less accurate than on the BBOB functions. Roughly counted, the model correctly predicts only 14 out of 24 of the functions for each of the two- and the five-dimensional cases. Interestingly, the models' predictions actually lay closer to the ground-truth column for the BBOB functions as to the simulated functions. This might indicate that the simulated functions were somewhat able to recreate landscapes with similar landscape features (hence the model prediction is working), but still so different that the algorithm performance was far off. Further analysis on the actual quality of the created test functions will be necessary to give a final conclusion to that argument.

\section{CONCLUSION}

To conclude this paper, we first discuss the initially posed contributions C-1 - C-3 individually. Finally, we summarize the primary outcome of this work with a more holistic view.

C-1 We introduced a framework for benchmarking parallel algorithms that are focused on solving computationally expensive to evaluate problems. The framework describes how to measure the actual evaluation costs that would occur on an expensive problem and how to transfer these costs to cheap functions. This procedure allows for rigorous performance analysis of algorithms as close to the actual evaluation case as possible.

The method is suited to deliver exact results for a given combination of available computational resources and objective function at hand. Instead of only benchmarking algorithms with singular specific batch sizes, the framework allows to directly measure the combined system performance of an algorithm and its function evaluation back end. The ability to rigorously judge the performance of an algorithm on a variety of landscapes before applying it to an expensive to evaluate function gives practitioners more concise choices without wasting costly evaluations.

Redefining the evaluation cost of algorithms to the actual cost in runtime that an evaluation would create on a given system allows the representation of the results in joined graphs. Convergence plots showing performance over time can show algorithms with different batch sizes combined on a single axis.

Additionally to a benchmarking method, we discussed the scarcity of expensive-to-evaluate real-world problems that are publicly available. We proposed the generation of data-driven test functions based on Kriging simulations to circumvent this problem. This extends methods that were previously described by Zaefferer and Rehbach [40]. The methods are now publicly available in the form of the R-package 'COBBS' (Continuous 
Optimization Benchmarks By Simulation). Thus, applying the method to new problems requires just a few lines of code.

The simulation instances arguably deliver far more abstraction between generated instances and also between the instances and the real function that the data was initially taken from. Depending on the application scenario, this can be beneficial or a drawback. For a suite of benchmarking problems, a diverse test set on which algorithms are compared can be beneficial. Algorithms can be compared on a broad set of landscapes with different properties. Yet, sometimes the goal is to recreate a real-world problem so that algorithms would show similar performance on the simulated case as they do on the real function. In such a case, the quality of the simulated functions has to be inspected carefully.

C-2 The described benchmarking framework was applied to judge the performance of the newly introduced ML-EI algorithm. The presented algorithm shows great overall performance, especially on multi-modal functions with adequate global structure. More importantly, it delivered robust results over a large set of different landscapes for expensive blackbox optimization. This makes the algorithm well applicable to unknown black-box types of problems. We assume that this is a positive outcome of the synergy between both the explorative and the exploitative parts of the algorithm. While the exploitative steps (1+1 ES and PV infill criterion) result in quick convergence on easier problem landscapes, the Q-EI phases help explore the landscapes of more complex or multimodal landscapes.

The results also indicate that the discussed problem of too much exploration in parallel model-based optimization can be circumvented. The presented performance of the Q-EI and IPI methods dropped significantly for higher batch sizes. For Q-EI, high batch sizes result in points mainly being placed in untouched search regions. With IPI, many similar points might be proposed if the batch size is too high. Both of these behaviors prohibit an efficient convergence to the optimum. In contrast to that, the ML-EI parallelization scaled well also with a batch size of 16 . There, it was never outperformed for any two-dimensional function and only on two of the five-dimensional functions. Similarly, good scaling was only observed by MOI-MBO.

Future research could be applied to a range of topics regarding the ML-EI algorithm. Firstly, the choice of the 1+1 ES as a local optimizer. Generally, any local optimizer could be used to replace the ES. Thus, additional research might lead to better alternatives. Secondly, the rate at which the search should switch from exploration to exploitation needs further investigation. Is the currently applied linear transition based on the remaining budget an ideal choice? Would an earlier or later transition to exploitation result in better performance, or can this transition even be guided based on certain landscape features? To give just one example, noticing that one is faced with a relatively simple unimodal problem, a quick transition to pure exploitation should yield the best performance.

C-3 The adaptive batch size variant of the ML-EI algorithm uses benchmark data from the previous algorithm runs, as well as landscape feature analysis in an application to predict efficient batch sizes on new functions. The described approach worked well for predicting new BBOB instances in a train and test split similar to the one employed by Mersmann et al. [26]. This is likely due to the fact that the instances in the BBOB suite are still rather similar to one another. As expected, the results on the simulated functions (which can be more argued to be true abstractions and unseen new problems) showed less predictive accuracy. Future work is required to see how results on new functions can be improved. Topics of interest might focus on choosing a different modeling technique or providing a larger and more diverse training data set. A promising idea to increase the model's predictive power is introduced in recent work by Muñoz and Smith-Miles [28]. They argue that each function can be represented in a multi-dimensional ELA feature space. Then, genetic programming is used to sample new functions that fill gaps in that search space. Having a sufficiently filled function space as the training data sample for the batch size prediction model might yield better results as less abstraction to new functions should be required from the prediction model.

In summary, we introduced a set of methods that synergize with each other in order to tackle multiple complexities of expensive to evaluate black-box problems. Firstly, we mitigate the high cost of such problems by introducing a sample efficient parallel optimizer that also scales well to high batch sizes for systems with large amounts of available CPU cores. The parallel algorithm can now be fairly compared through the introduced benchmarking framework. As discussed, tuning an algorithm's configuration is usually not possible for expensive problems. Therefore, the algorithm can be combined with the described online adaptation for reconfiguring batch sizes. Furthermore, the availability issue for real-world similar benchmarks is tackled with the publication of 'COBBS'. Issues of experiment reproducibility are bypassed by making all codes and experiments publicly available.

\section{ACKNOWLEDGMENTS}

This work is supported by the German Federal Ministry of Education and Research in the funding program Forschung an Fachhochschulen under the grant number 13FH007IB6. 


\section{REFERENCES}

[1] T. Bartz-Beielstein, C. Lasarczyk, and M. Preuss, "Sequential parameter optimization," in Proceedings Congress on Evolutionary Computation 2005 (CEC'05), Edinburgh, Scotland, 2005, p. 1553.

[2] T. Bartz-Beielstein, J. Stork, M. Zaefferer, M. Rebolledo, C. Lasarczyk, J. Ziegenhirt, W. Konen, O. Flasch, P. Koch, M. Friese, L. Gentile, and F. Rehbach, "Spot: Sequential parameter optimization toolbox - version 2.0.4," Online, 2019, available: https://cran.r-project.org/package=SPOT, accessed: 2019-11-15.

[3] T. Bartz-Beielstein and M. Zaefferer, "Model-based methods for continuous and discrete global optimization," Applied Soft Computing, vol. 55, pp. $154-167,2017$.

[4] B. Bischl, J. Richter, J. Bossek, D. Horn, J. Thomas, and M. Lang, mlrMBO: A Modular Framework for Model-Based Optimization of Expensive Black-Box Functions, 2017. [Online]. Available: http://arxiv.org/abs/1703.03373

[5] B. Bischl, S. Wessing, N. Bauer, K. Friedrichs, and C. Weihs, "Moimbo: multiobjective infill for parallel model-based optimization," in International Conference on Learning and Intelligent Optimization. Springer, 2014, pp. 173-186.

[6] L. Breiman, "Random forests," Machine learning, 2001.

[7] N. A. Cressie, Statistics for Spatial Data. New York, NY: Wiley, 1993.

[8] S. J. Daniels, A. A. Rahat, R. M. Everson, G. R. Tabor, and J. E. Fieldsend, "A suite of computationally expensive shape optimisation problems using computational fluid dynamics," in International Conference on Parallel Problem Solving from Nature. Springer, 2018, pp. 296-307.

[9] G. De Ath, R. M. Everson, J. E. Fieldsend, and A. A. M. Rahat, " $\varepsilon$ shotgun: $\varepsilon$-greedy batch bayesian optimisation," in Proceedings of the 2020 Genetic and Evolutionary Computation Conference, ser. GECCO '20. ACM, 2020, pp. 787-795.

[10] G. De Ath, R. M. Everson, A. A. Rahat, and J. E. Fieldsend, "Greed is good: Exploration and exploitation trade-offs in bayesian optimisation," ACM Transactions on Evolutionary Learning and Optimization, 2021.

[11] J. Derrac, S. García, D. Molina, and F. Herrera, "A practical tutorial on the use of nonparametric statistical tests as a methodology for comparing evolutionary and swarm intelligence algorithms," Swarm and Evolutionary Computation, vol. 1, no. 1, pp. 3-18, Mar. 2011.

[12] A. Forrester, A. Keane et al., Engineering design via surrogate modelling: a practical guide. John Wiley \& Sons, 2008.

[13] D. Ginsbourger, R. Le Riche, and L. Carraro, "Kriging is well-suited to parallelize optimization," in Computational intelligence in expensive optimization problems. Springer, 2010, pp. 131-162.

[14] R. T. Haftka, D. Villanueva, and A. Chaudhuri, "Parallel surrogateassisted global optimization with expensive functions-a survey," Structural and Multidisciplinary Optimization, vol. 54, no. 1, pp. 3-13, 2016.

[15] N. Hansen, A. Auger, O. Mersmann, T. Tusar, and D. Brockhoff, "COCO: A platform for comparing continuous optimizers in a black-box setting," ArXiv e-prints, Aug. 2016, arXiv ID: 1603.08785v3.

[16] N. Hansen, Y. Akimoto, and P. Baudis, "Cma-es/pycma on github," Feb. 2019. [Online]. Available: https://doi.org/10.5281/zenodo.2559635

[17] N. Hansen, S. Finck, R. Ros, and A. Auger, "Real-parameter blackbox optimization benchmarking 2009: Noiseless functions definitions," INRIA, Tech. Rep. RR-6829, 2009.

[18] M. Hollander, D. A. Wolfe, and E. Chicken, Nonparametric Statistical Methods, 3rd ed. New York, NY: Wiley, 2014.

[19] G. James, D. Witten, T. Hastie, and R. Tibshirani, An Introduction to Statistical Learning. Springer, 2017.

[20] D. R. Jones, M. Schonlau, and W. J. Welch, "Efficient global optimization of expensive black-box functions," Journal of Global optimization, vol. 13, no. 4, pp. 455-492, 1998.

[21] P. Kerschke and H. Trautmann, "Automated algorithm selection on continuous black-box problems by combining exploratory landscape analysis and machine learning," Evolutionary Computation, vol. 27, no. 1, pp. 99-127, Mar 2019.

[22] — "Comprehensive feature-based landscape analysis of continuous and constrained optimization problems using the r-package flacco," in Applications in Statistical Computing. Springer, 2019, pp. 93-123.

[23] H. Kotthaus, J. Richter, A. Lang, J. Thomas, B. Bischl, P. Marwedel, J. Rahnenführer, and M. Lang, "Rambo: Resource-aware model-based optimization with scheduling for heterogeneous runtimes and a comparison with asynchronous model-based optimization," in International Conference on Learning and Intelligent Optimization. Springer, 2017.

[24] M. D. McKay, R. J. Beckman, and W. J. Conover, "Comparison of three methods for selecting values of input variables in the analysis of output from a computer code," Technometrics, vol. 21, no. 2, 1979.
[25] O. Mersmann, B. Bischl, H. Trautmann, M. Preuss, C. Weihs, and G. Rudolph, "Exploratory landscape analysis," in Proceedings of the 13th annual conference on Genetic and evolutionary computation, 2011, pp. 829-836.

[26] O. Mersmann, M. Preuss, and H. Trautmann, "Benchmarking evolutionary algorithms: Towards exploratory landscape analysis," in Parallel Problem Solving from Nature, PPSN XI, 2010, pp. 73-82.

[27] K. Mullen, D. Ardia, D. Gil, D. Windover, and J. Cline, "DEoptim: An R package for global optimization by differential evolution," Journal of Statistical Software, vol. 40, no. 6, pp. 1-26, 2011.

[28] M. A. Muñoz and K. Smith-Miles, "Generating new space-filling test instances for continuous black-box optimization," Evolutionary computation, vol. 28, no. 3, pp. 379-404, 2020.

[29] N. V. Queipo, R. T. Haftka, W. Shyy, T. Goel, R. Vaidyanathan, and P. K. Tucker, "Surrogate-based analysis and optimization." Progress in aerospace sciences, 2005.

[30] M. Rebolledo, F. Rehbach, A. E. Eiben, and T. Bartz-Beielstein, "Parallelized bayesian optimization for expensive robot controller evolution," in PPSN XVI, 2020.

[31] I. Rechenberg, Evolutionsstrategie: Optimierung technischer Systeme nach Prinzipien der biologischen Evolution. Frommann-Holzboog, 1973.

[32] F. Rehbach, M. Zaefferer, B. Naujoks, and T. Bartz-Beielstein, "Expected improvement versus predicted value in surrogate-based optimization," in Proceedings of the 2020 Genetic and Evolutionary Computation Conference, ser. GECCO '20. ACM, 2020, pp. 868-876.

[33] F. Rehbach, M. Zaefferer, J. Stork, and T. Bartz-Beielstein, "Comparison of parallel surrogate-assisted optimization approaches," in Proceedings of the Genetic and Evolutionary Computation Conference - GECCO 18. ACM Press, 2018, pp. 1348-1355.

[34] O. Roustant, D. Ginsbourger, and Y. Deville, "DiceKriging, DiceOptim: Two R packages for the analysis of computer experiments by krigingbased metamodeling and optimization," Journal of Statistical Software, vol. 51, no. 1, pp. 1-55, 2012.

[35] M. Schonlau, "Computer experiments and global optimization," Ph.D. dissertation, University of Waterloo, 1997.

[36] R. K. Ursem, From Expected Improvement to Investment Portfolio Improvement: Spreading the Risk in Kriging-Based Optimization. Cham: Springer International Publishing, 2014, pp. 362-372.

[37] K. Weinert, J. Mehnen, and G. Rudolph, "Dynamic neighborhood structures in parallel evolution strategies," Complex Systems, vol. 13, no. 3, pp. 227-244, 2001

[38] S. Wessing and M. Preuss, "The true destination of EGO is multilocal optimization," in 2017 IEEE Latin American Conference on Computational Intelligence (LA-CCI). IEEE, nov 2017.

[39] M. Zaefferer, A. Fischbach, B. Naujoks, and T. Bartz-Beielstein, "Simulation based test functions for optimization algorithms," in Proceedings of the Genetic and Evolutionary Computation Conference 2017. Berlin, Germany: ACM, july 2017, p. 8.

[40] M. Zaefferer and F. Rehbach, "Continuous optimization benchmarks by simulation," in Parallel problem solving from nature - PPSN XVI: 16th international conference, 2020. 